Bull. Mater. Sci., Vol. 7, No. 1, March 1985, pp. 63-69. (C) Printed in India.

\title{
Electrical, electromechanical and thermal properties of thin tellurium films
}

\author{
S SAMPATH and KOLLURI V RAMANAIAH \\ Department of Physics, PSG College of Technology, Coimbatore 641 004, India \\ MS received 1 June 1984; revised 25 October 1984
}

\begin{abstract}
Thin films of tellurium of wide range of thicknesses have been deposited by vacuum evaporation and their electrical properties such as electrical resistivity and temperature coefficient of resistance have been measured. The suitability of these films for possible use as strain gauges has been studied and their strain resistivity behaviour is presented. The thermal conductivity of these films have been determined and these results are presented alongwith. An interesting phenomenon has been noticed. In all these effects an extraordinary behaviour is observed at a specific thickness. This smears out with an increase in the thickness of the film. These effects are explained in terms of size effects in thin films.
\end{abstract}

Keywords. Tellurium films; electrical properties; thermal properties; strain gauge.

\section{Introduction}

Tellurium forms compounds with $\mathrm{Bi}, \mathrm{Mg}, \mathrm{Sn}, \mathrm{Cd}$ and $\mathrm{Hg}$. These compounds are known as tellurides and are given the general name 'chalcogenides'. The energy gaps in them are generally large and the presence of impurities leads to interesting and important luminescent and photoconductive effects.

A study of the electrical and thermal properties of tellurium will help in the study and interpretation of the properties of thin films of these tellurides. For example, many of the tellurides are piezo-electric. A thin film tellurium strain gauge may possess sensitivities much greater than are known at present. Tellurides are used to detect and measure visible radiation. Hence compounds of tellurium offer vast scope for further work.

\section{Experimental techniques}

Thin films of tellurium of wide range of thicknesses were prepared on mica and mylar substrates kept at room temperature by evaporating different quantities of tellurium from a molybdenum boat (Kasturi 1962) at a pressure of $5 \times 10^{-5}$ torr. For all evaporations the source to substrate distance was kept constant. The thickness of these films were determined using a multiple beam interferometer.

\section{Measurement of resistivity}

These measurements were carried out at room temperature of $27^{\circ} \mathrm{C}$ for seven samples, having thicknesses ranging from $875 \AA$ to $4275 \AA$ using a resistance bridge having an accuracy of $2 \%$. Figure 1 relates the electrical resistivity with thickness. 


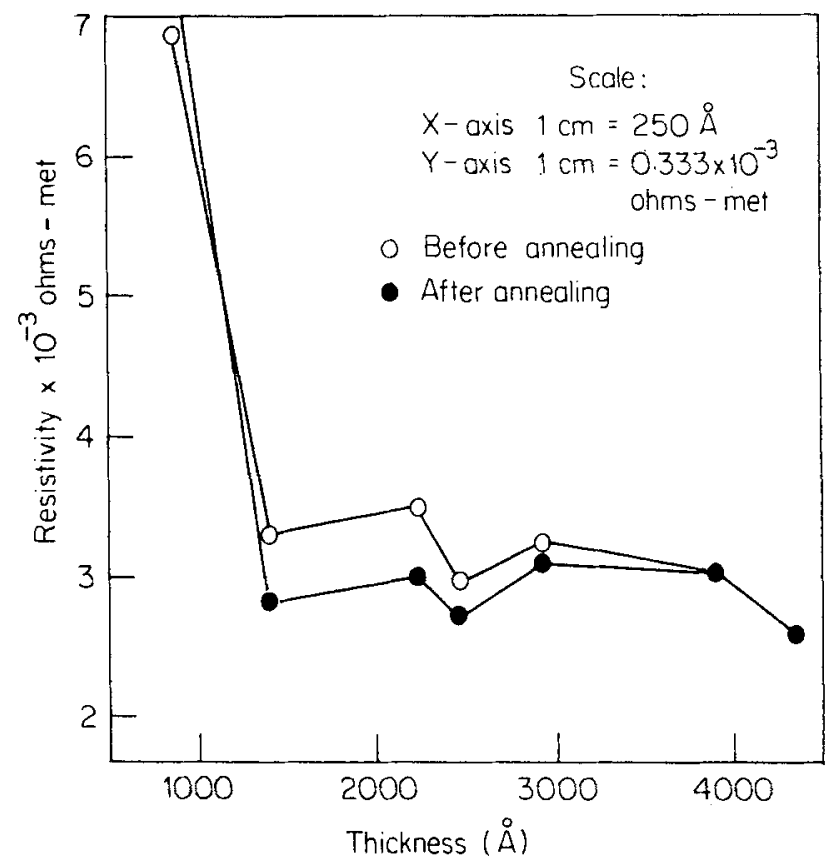

Figure 1. Variation of resistivity with thickness.

\section{Measurement of TCR}

For this measurement, all the seven samples were mounted on a substrate holder and kept inside a vacuum chamber and the required connecting leads were taken through electrical feed-throughs. The pressure inside the chamber was maintained at $10^{-3}$ torr during the measurement to prevent any possible effects of oxidation. The films were heated to the desired temperature, again in the chamber itself, using an internally mounted radiant heater and the temperature of these films were measured by using copper-constantan thermocouples. The change in resistance of these films were measured using a resistance bridge. Figure 2 relates the TCR with thickness of the film and temperature and figure 3 relates the TCR with temperature for a particular thickness.

\section{Electromechanical behaviour}

The strain resistivity behaviour of these films were studied using the cantilever technique (Sampath 1981) as shown in figure 4. The film on the flexible substrate is cemented firmly to a mild steel bar $(2.5 \mathrm{~cm}$ wide, $0.5 \mathrm{~cm}$ thick and $100 \mathrm{~cm}$ in length) such that the distance between the centre of the gauge to the point of application of the load at the other end of the beam is $75 \mathrm{~cm}$. The mild steel beam is firmly clamped to a rigid support as a single cantilever and a weight pan is fixed at the other end of the beam. The change in resistance of the gauge with load is measured by connecting it to a digital ohm-meter. Weights are added in steps and the changes in resistance are observed both while loading and unloading. The 'hysteresis behaviour' which is defined as the 


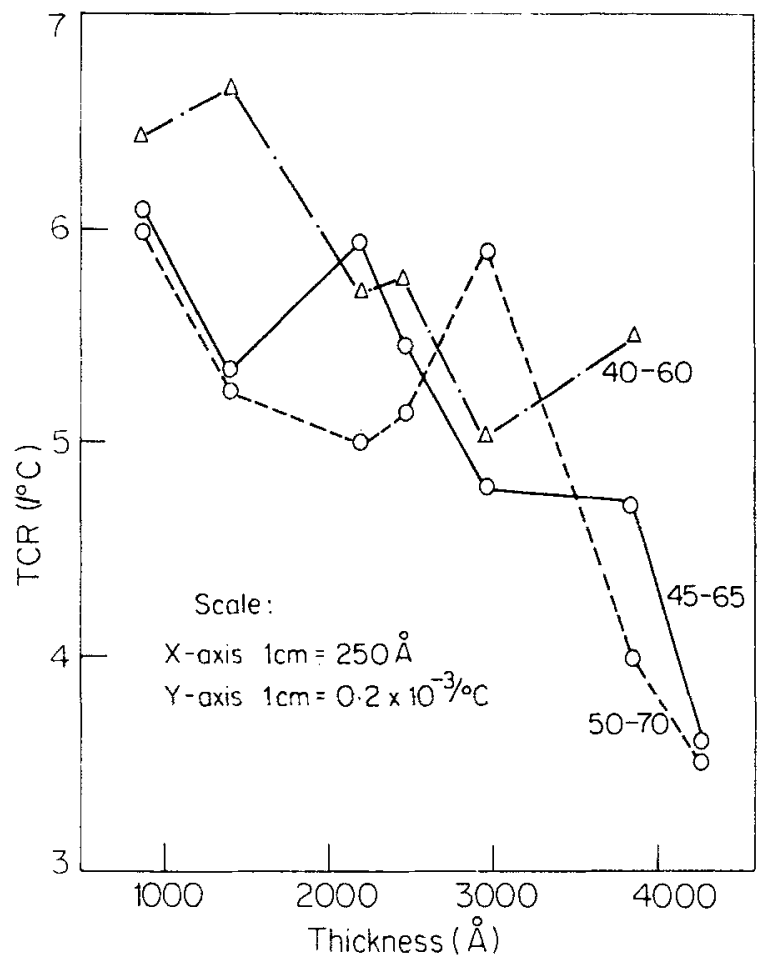

Figure 2. Variation of TCR with thickness.

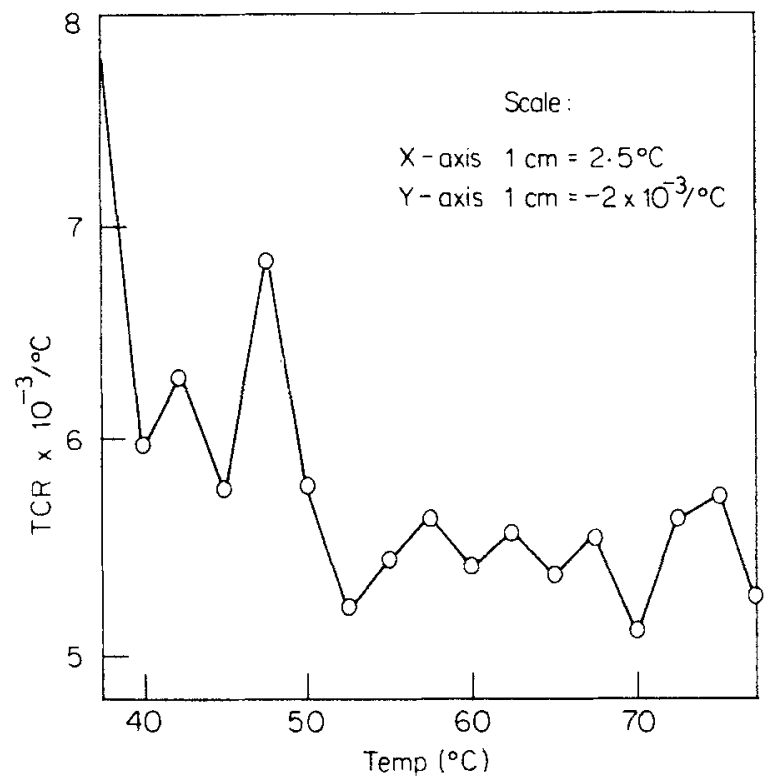

Figure 3. Variation of TCR with temperature for a particular thickness. 


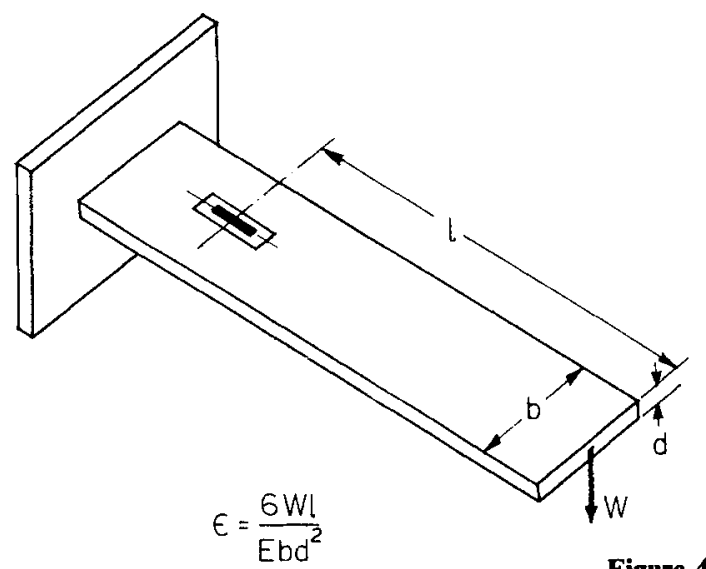

Figure 4. Strain gauge bonded to a cantilever system.

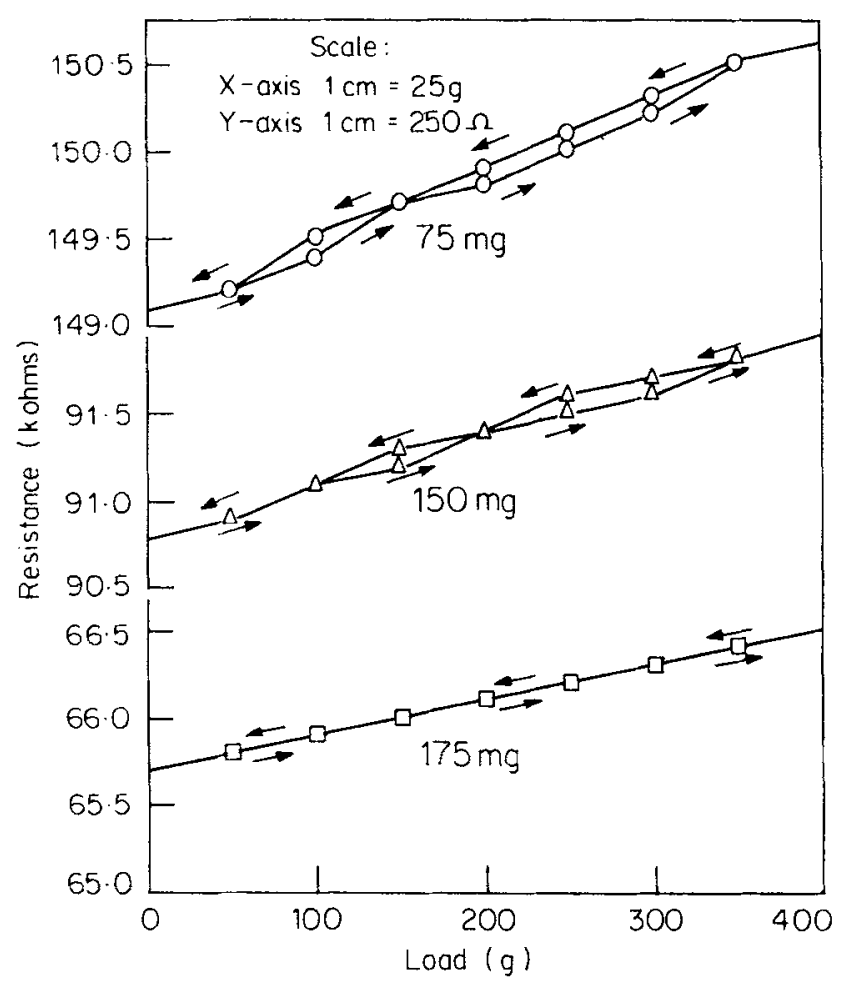

Figure 5. Hysteresis effect.

phenomenon where the values of resistance indicated for 'ascending' and 'descending' strain of the gauge are not exactly equal, has been studied for these films and the results are shown in figure 5 .

The gauge factor $G$ of these films were determined using the formula

$$
G=\frac{E b d^{2}}{6 W l} \cdot \frac{\Delta R}{R}
$$

where $E$ is the Young's modulus of the material of the beam, $w$ the load applied, $l$ 


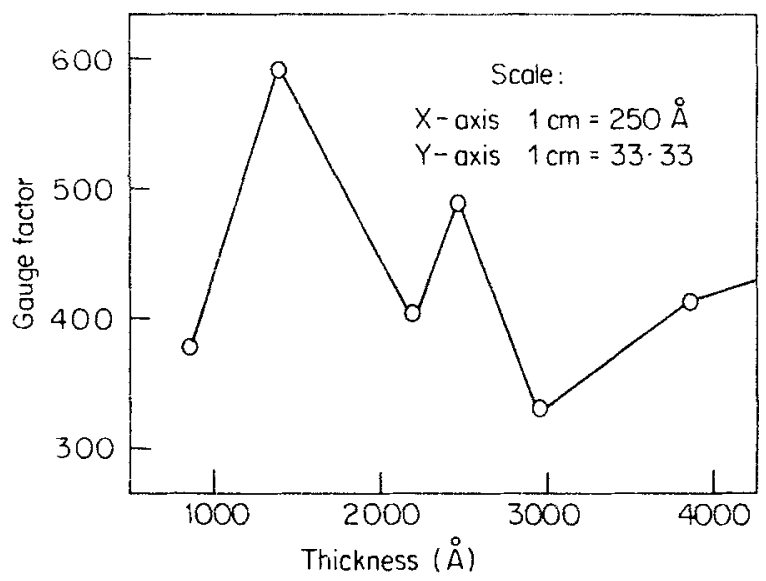

Figure 6. Variation of gauge factor with thickness.

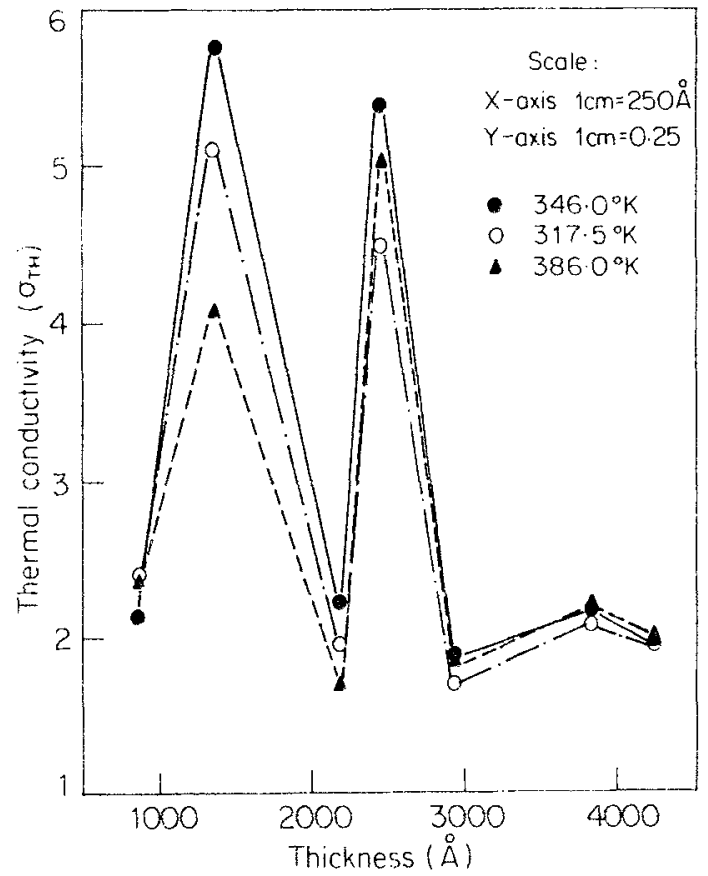

Figure 7. Variation of thermal conductivity with thickness.

distance between the centre of the gauge and the point of application of the load, $R$ the initial resistance and $\Delta R$ the change in resistance of the gauge for a given load. The variation of gauge factor with thickness is shown in figure 6 .

\section{Thermal conductivity}

The thermal conductivity of these films were determined in situ by a method developed by Nath and Chopra (1973). The thermal conductivity of these films for three different 
temperature ranges have been determined. Figure 7 shows the variation of thermal conductivity with thickness and temperature.

\section{Discussion}

The electrical resistivity of tellurium films shows an oscillatory behaviour for all thicknesses. The electrical resistivity shows a steep fall as the thickness increases from 850 to $1412 \AA$ and later shows an oscillatory behaviour (figure 1). This behaviour is observed both for annealed and unannealed films. In general the resistivity of the films decreased with annealing and the oscillatory behaviour also appears to be reduced. Annealing was done at $80^{\circ} \mathrm{C}$ for $1 \mathrm{hr}$ and the entire operation was carried out in situ. The annealing has not been carried out at higher temperatures since the films have low evaporation temperature. The pronounced maxima and minima occur for specific thicknesses in both cases.

The observed phenomenon can be qualitatively accounted for by the following arguments. The high resistance at very low thicknesses can be attributed to the large inter-island separation in the film. When the thickness is increased the inter-island gap gets reduced and thereby decreases the resistance. This is further supported by the fact that the resistance further reduces on annealing. Further increase in thickness may introduce different amounts of defects in the film and also a change in the structure of the film. This results in an oscillatory behaviour of the resistivity. As the thickness is increased beyond $3000 \AA$, all these structural and defect contributions even out and the oscillatory behaviour reduces. These arguments are further supported by a similar behaviour observed in thermal conductivity measurements also. A similar result for tellurium has been reported by Chaudhuri (1974) and he has attributed this to the variation of structure with thickness.

The TCR of thin films of tellurium is found for three ranges $40-60^{\circ} \mathrm{C}, 45-65^{\circ} \mathrm{C}$, $50-70^{\circ} \mathrm{C}$ for thicknesses ranging from $850 \AA$ to $4200 \mathrm{~A}$. It is found to be negative which shows that tellurium belongs to a class of semi-metals. The TCR also shows an oscillatory behaviour with thickness of the film (figure 2). An explanation for this behaviour can be based on the same arguments as given above for the resistance variation. In general as the resistivity of a material decreases, the TCR decreases. The variation of TCR with thickness mainly depends on the resistivity of the film. The variation of $\mathrm{TCR}$ with temperature for a particular thickness is anomalous. This can be accounted for only by assuming that the activation energy of the electron varies with temperature particularly in a polycrystalline film.

The hysteresis effect decreases appreciably after a few cycles of loading and unloading and the gauge is able to follow the strain reversibly after a few cycles (figure 5). This may be due to the relief of strain in the film itself after a few cycles. The variation of gauge factor is shown in figure (6). This property also shows an oscillatory behaviour with thickness. This could be accounted for on the basis of the structure of the film which seems to vary with thickness. The gauge factor is also intimately connected with inter-island separation. It is interesting to note that a gauge factor as high as 590 is obtained for a thickness of 1412 A. This is a significant result.

The thermal conductivity of tellurium films also shows an oscillatory behaviour with thickness as shown in figure (7) and shows a peak value at thicknesses of 1412 and $2474 \AA$. The explanation for it could be along the same lines as that of the electrical 
resistivity. As can be seen from figure (7) the thermal conductivity oscillates as temperature increases for all thicknesses of the film. A peak value of $5 \cdot 7 \mathrm{Wm}^{-1} \mathrm{~K}^{-1}$ is obtained for a thickness of $1412 \AA$. Fisher et al (1961) have determined the thermal conductivity of bulk tellurium over a range of temperatures. The thermal conductivity varies over a wide range of values. This variation is attributed to phonon-phonon scattering. No conclusive theory is yet available to account for the variation of thermal conductivity in thin tellurium films.

\section{g. Conclusion}

The electrical conductivity, thermal conductivity, TCR and gauge factor determined in thin films of tellurium shows that there is an oscillatory behaviour in these properties with thickness. It is also been observed that the maxima and minima of these oscillations very nearly coincide with respect to the thickness for all the properties. While no conclusive explanation can be given for the same it could be qualitatively interpreted as due to the variation of defect concentration and structure with thickness. This opinion seems to be supported by earlier reports of Chaudhuri and others. An extraordinarily high gauge factor has been observed for a thickness of $1412 \AA$ for which also there is no immediate explanation available.

\section{Acknowiedgements}

The authors thank Prof. G R Damodaran and $D_{i} F_{k}$ Venkataraman, for providing facilities. They also wish to acknowledge the assistance rendered by $\mathrm{Mr} \mathrm{R}$ Palanisamy.

\section{References}

Chaudhuri A K 1974 Indian J. Pure Appl. Phys. 12339

Fisher et al 1961 Thermal conduction in semiconductors (eds) J R Drabble and H J Goldsmid (New York: Pergamon Press)

Kasturi L. C 1962 Thin flm phenomena (New York: McGraw Hill)

Premnath and Chopra K.L. 1973 Thin Solid Films 1829

Sampath $\mathrm{S} 1981$ Electroniechanical and thermal properties of thin films $\mathrm{Ph} . \mathrm{D}$. Thesis, University of Madras, India 